PROCEEDINGS OF THE

AMERICAN MATHEMATICAL SOCIETY

Volume 136, Number 11, November 2008, Pages 3975-3981

S 0002-9939(08)09444-6

Article electronically published on June 5, 2008

\title{
EXISTENCE OF QUASI-ARCS
}

\author{
JOHN M. MACKAY
}

(Communicated by Mario Bonk)

\begin{abstract}
We show that doubling, linearly connected metric spaces are quasiarc connected. This gives a new and short proof of a theorem of Tukia.
\end{abstract}

\section{INTRODUCTION}

It is a standard topological fact that a complete metric space which is locally connected, connected and locally compact is arc-wise connected. Tukia [6] showed that an analogous geometric statement is true: if a complete metric space is linearly connected and doubling, then it is connected by quasi-arcs, quantitatively. In fact, he proved a stronger result: any arc in such a space may be approximated by a local quasi-arc in a uniform way. In this paper we give a new and more direct proof of this fact.

This result is of interest in studying the quasi-symmetric geometry of metric spaces. Such geometry arises in the study of the boundaries of hyperbolic groups; Tukia's result was used in this context by Bonk and Kleiner [1, and also by the author [5]. (Bonk and Kleiner used Assouad's embedding theorem to translate Tukia's result from its original context of subsets of $\mathbb{R}^{n}$ into our setting of doubling and linearly connected metric spaces.)

Before stating the theorem precisely, we recall some definitions. A metric space $(X, d)$ is said to be doubling if there exists a constant $N$ such that every ball can be covered by at most $N$ balls of half the radius. Note that any complete, doubling metric space is proper: all closed balls are compact.

We say $(X, d)$ is L-linearly connected for some $L \geq 1$ if for all $x, y \in X$ there exists a compact, connected set $J \ni x, y$ of diameter less than or equal to $L d(x, y)$. (This is also known as bounded turning or LLC(1).) We can actually assume that $J$ is an arc at the cost of increasing $L$ by an arbitrarily small amount. To see this, note that $X$ is locally connected, and so the connected components of an open set are open. Thus, for any open neighborhood $U$ of $J$, the connected component of $U$ that contains $J$ is an open set. We can replace $J$ inside $U$ by an arc with the same endpoints, since any open, connected subset of a locally compact, locally connected metric space is arc-wise connected [3. Corollary 32.36].

For any $x$ and $y$ in an embedded arc $A$, we denote by $A[x, y]$ the closed, possibly trivial, subarc of $A$ that lies between them. We say that an arc $A$ in a doubling

Received by the editors October 17, 2007.

2000 Mathematics Subject Classification. Primary 30C65; Secondary 54D05.

Key words and phrases. Quasi-arc, linearly connected, bounded turning.

This research was partially supported by NSF grant DMS-0701515.

(C)2008 American Mathematical Society 3975

Reverts to public domain 28 years from publication 
and complete metric space is an $\epsilon$-local $\lambda$-quasi-arc if $\operatorname{diam}(A[x, y]) \leq \lambda d(x, y)$ for all $x, y \in A$ such that $d(x, y) \leq \epsilon$. (This terminology is explained by Tukia and Väisälä's characterization of quasi-symmetric images of the unit interval as those metric arcs that are doubling and bounded turning [7].)

One non-standard definition will be useful in our exposition. We say that an arc $B \epsilon$-follows an arc $A$ if there exists a coarse map $p: B \rightarrow A$, sending endpoints to endpoints, such that for all $x, y \in B, B[x, y]$ is in the $\epsilon$-neighborhood of $A[p(x), p(y)]$; in particular, $p$ displaces points at most $\epsilon$. (We call the map $p$ coarse to emphasize that it is not necessarily continuous.)

The condition that $B \epsilon$-follows $A$ is stronger than the condition that $B$ is contained in the $\epsilon$-neighborhood of $A$. It says that, coarsely, the arc $B$ can be obtained from the $\operatorname{arc} A$ by cutting out 'loops.' (Of course, $A$ contains no actual loops, but it may have subarcs of a large diameter whose endpoints are $2 \epsilon$-close.)

We can now state the stronger version of Tukia's theorem precisely, and as an immediate corollary our initial statement [6, Theorem 1B, Theorem 1A]:

Theorem 1.1 (Tukia). Suppose $(X, d)$ is an L-linearly connected, $N$-doubling, complete metric space. For every arc $A$ in $X$ and every $\epsilon>0$, there is an arc $J$ that $\epsilon$-follows $A$, has the same endpoints as $A$, and is an $\alpha \epsilon$-local $\lambda$-quasi-arc, where $\lambda=\lambda(L, N) \geq 1$ and $\alpha=\alpha(L, N)>0$.

Corollary 1.2 (Tukia). Every pair of points in an L-linearly connected, $N$-doubling, complete metric space is connected by a $\lambda$-quasi-arc, where $\lambda=\lambda(L, N) \geq 1$.

Our strategy for proving Theorem 1.1 is straightforward: find a method of straightening an arc on a given scale (Proposition 2.1), then apply this result on a geometrically decreasing sequence of scales to get the desired local quasi-arc as a limiting object. The statement of this proposition and the resulting proof of the theorem essentially follow Tukia [6], but the proof of the proposition is new and much shorter. We include a complete proof for the convenience of the reader.

The author thanks Mario Bonk and, in particular, his advisor, Bruce Kleiner, for many helpful suggestions and fruitful conversations.

\section{MAIN RESULTS}

Given any $\operatorname{arc} A$ and $\iota>0$, the following proposition allows us to straighten $A$ on a scale $\iota$ inside the $\iota$-neighborhood of $A$.

Proposition 2.1. Given a complete metric space $X$ that is L-linearly connected and $N$-doubling, there exist constants $s=s(L, N)>0$ and $S=S(L, N)>0$ with the following property: for each $\iota>0$ and each arc $A \subset X$, there exists an arc $J$ that $\iota$-follows $A$, has the same endpoints as $A$, and satisfies

$$
\forall x, y \in J, d(x, y)<s \iota \Longrightarrow \operatorname{diam}(J[x, y])<S \iota .
$$

We will apply this proposition on a decreasing sequence of scales to get a local quasi-arc in the limit. The key step in proving this is given by the following lemma.

Lemma 2.2. Suppose $(X, d)$ is an L-linearly connected, $N$-doubling, complete metric space, and let $s, S, \epsilon$ and $\delta$ be fixed positive constants satisfying $\delta \leq$ $\min \left\{\frac{s}{4+2 S}, \frac{1}{10}\right\}$. Now, if we have a sequence of arcs $J_{1}, J_{2}, \ldots, J_{n}, \ldots$ in $X$, such that for every $n \geq 1$

$$
\text { - } J_{n+1} \epsilon \delta^{n} \text {-follows } J_{n} \text {, and }
$$


- $J_{n+1}$ satisfies (因) with $\iota=\epsilon \delta^{n}$ and $s, S$ as fixed above,

then the Hausdorff limit $J=\lim _{\mathcal{H}} J_{n}$ exists and is an $\epsilon \delta^{2}$-local $\frac{4 S+3 \delta}{\delta^{2}}$-quasi-arc.

Moreover, the endpoints of $J_{n}$ converge to the endpoints of $J$, and $J$-follows $J_{1}$.

We shall need some standard definitions. The (infimal) distance between two subsets $U, V \subset X$ is defined as $d(U, V)=\inf \{d(u, v): u \in U, v \in V\}$. If $U=\{u\}$, then we set $d(u, V)=d(U, V)$.

The $r$-neighborhood of $U$ is the set $N(U, r)=\{x: d(x, U)<r\}$, and the Hausdorff distance between $U$ and $V, d_{\mathcal{H}}(U, V)$, is defined to be the infimal $r$ such that $U \subset N(V, r)$ and $V \subset N(U, r)$. For more information, see [2, Chapter 7].

We will now prove Theorem 1.1 .

Proof of Theorem 1.1. Let $s$ and $S$ be given by Proposition 2.1, and set $\delta=$ $\min \left\{\frac{s}{4+2 S}, \frac{1}{10}\right\}$.

Let $J_{1}=A$ and apply Proposition 2.1 to $J_{1}$ and $\iota=\epsilon \delta$ to get an arc $J_{2}$ that $\epsilon \delta$-follows $J_{1}$. Repeat, applying the lemma to $J_{n}$ and $\iota=\epsilon \delta^{n}$, to get a sequence of $\operatorname{arcs} J_{n}$, where each $J_{n+1} \epsilon \delta^{n}$-follows $J_{n}$ and satisfies (図) with $\iota=\epsilon \delta^{n}$.

We can now apply Lemma 2.2 to find an $\alpha \epsilon$-local $\lambda$-quasi-arc $J$ that $\epsilon$-follows $A$, where $\alpha=\delta^{2}$ and $\lambda=\frac{4 S+3 \delta}{\delta^{2}}$. Every $J_{n}$ has the same endpoints as $A$, so $J$ will also have the same endpoints.

The proof of Lemma 2.2 relies on some fairly straightforward estimates and a classical characterization of an arc.

Proof of Lemma 2.2. For every $n \geq 1, J_{n+1} \epsilon \delta^{n}$-follows $J_{n}$. We denote the associated coarse map by $p_{n+1}: J_{n+1} \rightarrow J_{n}$.

In the following, we will make frequent use of the inequality $\sum_{n=0}^{\infty} \delta^{n}<\frac{11}{9}$.

We begin by showing that the Hausdorff limit $J=\lim _{\mathcal{H}} J_{n}$ exists. The collection of all compact subsets of a compact metric space, given the Hausdorff metric, is itself a compact metric space [2, Theorem 7.3.8]. Since $\left\{J_{n}\right\}$ is a sequence of compact sets in a bounded region of a proper metric space, to show that the sequence converges with respect to the Hausdorff metric, it suffices to show that the sequence is Cauchy.

One bound follows by construction: $J_{n+m} \subset N\left(J_{n}, \frac{11}{9} \epsilon \delta^{n}\right)$ for all $m \geq 0$. For the second bound, take $J_{n+m}$ and split it into subarcs of diameter at most $\epsilon \delta^{n}$, and write this as $J_{n+m}=J_{n+m}\left[z_{0}, z_{1}\right] \cup \cdots \cup J_{n+m}\left[z_{k-1}, z_{k}\right]$ for some $z_{0}, \ldots, z_{k}$ and some $k>0$. Our coarse maps compose to give $p: J_{n+m} \rightarrow J_{n}$, showing that $J_{n+m}$ $\frac{11}{9} \epsilon \delta^{n}$-follows $J_{n}$. Furthermore, since $d\left(z_{i}, z_{i+1}\right) \leq \epsilon \delta^{n}$, we have $d\left(p\left(z_{i}\right), p\left(z_{i+1}\right)\right) \leq$ $4 \epsilon \delta^{n} \leq s \epsilon \delta^{n-1}$. Combining this with the fact that $p$ maps endpoints to endpoints, for $n \geq 2$ we have

$$
\begin{aligned}
J_{n} & =J_{n}\left[p\left(z_{0}\right), p\left(z_{1}\right)\right] \cup \cdots \cup J_{n}\left[p\left(z_{k-1}\right), p\left(z_{k}\right)\right] \subset N\left(\left\{p\left(z_{0}\right), \ldots, p\left(z_{k}\right)\right\}, S \epsilon \delta^{n-1}\right) \\
& \subset N\left(J_{n+m}, \frac{11}{9} \epsilon \delta^{n}+S \epsilon \delta^{n-1}\right) .
\end{aligned}
$$

Taken together, these bounds give $d_{\mathcal{H}}\left(J_{n}, J_{n+m}\right) \leq \frac{11}{9} \epsilon \delta^{n}+S \epsilon \delta^{n-1}$, so $\left\{J_{n}\right\}$ is Cauchy and the $\operatorname{limit} J=\lim _{\mathcal{H}} J_{n}$ exists. Moreover, $J$ is compact (by definition) and connected (because each $J_{n}$ is connected).

Now we let $a_{n}, b_{n}$ denote the endpoints of $J_{n}$. Since $p_{n}\left(a_{n}\right)=a_{n-1}$, and $p_{n}$ displaces points at most $\epsilon \delta^{n}$, the sequence $\left\{a_{n}\right\}$ is Cauchy and hence converges to some point $a \in J$. Similarly, $\left\{b_{n}\right\}$ converges to a point $b \in J$. 
There are two cases to consider. If $a=b$, then $d\left(a_{n}, b_{n}\right) \leq 2 \frac{11}{9} \epsilon \delta^{n} \leq s \epsilon \delta^{n-1}$. Consequently, $\operatorname{diam}\left(J_{n}\right) \leq S \epsilon \delta^{n-1}, J=\lim _{\mathcal{H}} J_{n}$ has diameter zero, and thus $J=$ $\{a\}$. Otherwise, $a \neq b$ and so $J$ is non-trivial. We claim that in this case $J$ is a local quasi-arc.

To show $J$ is an arc with endpoints $a$ and $b$ it suffices to demonstrate that every point $x \in J \backslash\{a, b\}$ is a cut point [4, Theorems 2-18 and 2-27]. The topology of $J_{n}$ induces an order on $J_{n}$ with least element $a_{n}$ and greatest element $b_{n}$. Given $x \in J$, we define three points $h_{n}(x), x_{n}$ and $t_{n}(x)$ that satisfy $a_{n}<h_{n}(x)<x_{n}<$ $t_{n}(x)<b_{n}$, where $x_{n}$ is chosen such that $d\left(x, x_{n}\right) \leq \frac{11}{9} \epsilon \delta^{n}$, and $h_{n}(x)$ and $t_{n}(x)$ are the first and last elements of $J_{n}$ at distance $(S+1) \epsilon \delta^{n-1}$ from $x$. As long as $x$ is not equal to $a$ or $b$, for $n$ greater than some $n_{0}$ these points will exist and this definition will be valid.

We shall denote the $\frac{11}{9} \epsilon \delta^{n}$-neighborhoods of $J_{n}\left[a_{n}, h_{n}(x)\right]$ and $J_{n}\left[t_{n}(x), b_{n}\right]$ by $H_{n}(x)$ and $T_{n}(x)$, respectively, and define $H(x)=\bigcup\left\{H_{n}(x): n \geq n_{0}\right\}$ (the Head) and $T(x)=\bigcup\left\{T_{n}(x): n \geq n_{0}\right\}$ (the Tail). By definition, $H(x)$ and $T(x)$ are open. We claim that, in addition, they are disjoint and cover $J \backslash\{x\}$, and so $x$ is a cut point.

Fix $y \in J$, and suppose $y \notin H(x) \cup T(x)$. We want to show that $y=x$. To this end, we bound the diameter of $J_{n}\left[h_{n}(x), t_{n}(x)\right]$ using $J_{n-1}$. Because $d\left(p_{n}\left(h_{n}(x)\right), p_{n}\left(t_{n}(x)\right)\right) \leq 2 \epsilon \delta^{n-1}+2(S+1) \epsilon \delta^{n-1} \leq s \epsilon \delta^{n-2}$, we know that the diameter of $J_{n-1}\left[p_{n}\left(h_{n}(x)\right), p_{n}\left(t_{n}(x)\right)\right]$ must be less than $S \epsilon \delta^{n-2}$. Thus the diameter of $J_{n}\left[h_{n}(x), t_{n}(x)\right]$ is less than $S \epsilon \delta^{n-2}+2 \epsilon \delta^{n-1}$, as $J_{n} \epsilon \delta^{n-1}$-follows $J_{n-1}$.

For every $n \geq n_{0}, y$ is $\frac{11}{9} \epsilon \delta^{n}$ close to some $y_{n} \in J_{n}$. Since $y \notin H(x) \cup T(x), y_{n}$ must lie in $J_{n}\left[h_{n}(x), t_{n}(x)\right]$, so

$$
\begin{aligned}
d(x, y) & \leq d\left(x, J_{n}\left[h_{n}(x), t_{n}(x)\right]\right)+\operatorname{diam}\left(J_{n}\left[h_{n}(x), t_{n}(x)\right]\right)+d\left(y_{n}, y\right) \\
& \leq 2 \frac{11}{9} \epsilon \delta^{n}+(S+2 \delta) \epsilon \delta^{n-2}=\left(2 \frac{11}{9} \delta^{2}+S+2 \delta\right) \epsilon \delta^{n-2} ;
\end{aligned}
$$

therefore $d(x, y)=0$ and $J \backslash(H(x) \cup T(x))=\{x\}$.

We now show that $H(x)$ and $T(x)$ are disjoint. If not, then $H_{n}(x) \cap T_{m}(x) \neq \emptyset$ for some $n$ and $m$. It suffices to assume $n \leq m$. Now $T_{m}(x) \subset N\left(J_{m}\left[x_{m}, b_{m}\right], \frac{11}{9} \epsilon \delta^{m}\right)$ by definition. We send $J_{m}$ to $J_{n}$ using $f=p_{n+1} \circ \cdots \circ p_{m}: J_{m} \rightarrow J_{n}$, to get that $T_{m}(x) \subset N\left(J_{n}\left[f\left(x_{m}\right), b_{n}\right], 3 \epsilon \delta^{n}\right)$. Since

$$
d\left(f\left(x_{m}\right), x_{n}\right) \leq d\left(f\left(x_{m}\right), x_{m}\right)+d\left(x_{m}, x\right)+d\left(x, x_{n}\right)<4 \epsilon \delta^{n}<s \epsilon \delta^{n-1}
$$

we have, even for $n=m$,

$$
T_{m}(x) \subset N\left(J_{n}\left[x_{n}, b_{n}\right], 3 \epsilon \delta^{n}\right) \cup B\left(x_{n},(S+3 \delta) \epsilon \delta^{n-1}\right) .
$$

Since $(S+3 \delta) \epsilon \delta^{n-1}+\frac{11}{9} \epsilon \delta^{n}<\left(S+\frac{1}{2}\right) \epsilon \delta^{n-1}, H_{n}(x)$ cannot meet $T_{m}(x)$ in the ball $B\left(x_{n},(S+3 \delta) \epsilon \delta^{n-1}\right)$. Thus $H_{n}(x) \cap T_{m}(x) \neq \emptyset$ implies that there exist points $p$ and $q$ in $J_{n}$ such that $a_{n} \leq p \leq h_{n}(x)<x_{n} \leq q \leq b_{n}$ and $d(p, q)<3 \epsilon \delta^{n}<s \epsilon \delta^{n-1}$. But then we know that $J_{n}[p, q]$ has diameter less than $S \epsilon \delta^{n-1}$, while containing both $h_{n}(x)$ and $x_{n}$. This contradicts the definition of $h_{n}(x)$, so $H(x) \cap T(x)=\emptyset$.

We have shown that $J$ is an arc with endpoints $a$ and $b$; it remains to show that $J$ is a local quasi-arc with the required constants.

Say we are given $x$ and $y$ in $J$, with $x_{n}$ and $y_{n}$ as before. Our argument shows that the segments $J_{n}\left[x_{n}, y_{n}\right]$ converge to some arc $\tilde{J}[x, y]$, because $J_{n+1}\left[x_{n+1}, y_{n+1}\right]$ $\left(\epsilon \delta^{n}+S \epsilon \delta^{n-1}\right)$-follows $J_{n}\left[x_{n}, y_{n}\right]$ for all $n \geq 2$. This arc $\tilde{J}[x, y]$ must lie in $J$; therefore $\tilde{J}[x, y]$ must equal $J[x, y]$. Now, suppose that $d(x, y) \in\left(\epsilon \delta^{n+1}, \epsilon \delta^{n}\right]$ holds 
for some $n \geq 2$. Then $d\left(x_{n}, y_{n}\right) \leq 3 \epsilon \delta^{n}+\epsilon \delta^{n}<s \epsilon \delta^{n-1}$, and so the subarc $J[x, y]$, which lies in $N\left(J_{n}\left[x_{n}, y_{n}\right], \frac{11}{9} \epsilon\left(\delta^{n}+S \delta^{n-1}\right)\right)$, has diameter less than

$$
S \epsilon \delta^{n-1}+3 \epsilon\left(\delta^{n}+S \delta^{n-1}\right) \leq \frac{4 S+3 \delta}{\delta^{2}} d(x, y)
$$

as desired.

Furthermore, this same argument gives that, for all $n \geq 2, J \frac{11}{9} \epsilon\left(\delta^{n}+S \delta^{n-1}\right)$ follows $J_{n}$, which itself $\frac{11}{9} \epsilon \delta$-follows $J_{1}=A$. Taking $n$ sufficiently large, we have that $J \in$-follows $A$.

\section{Discrete paths AND the Proof of Proposition 2.1}

The proof of Proposition 2.1 is based on a quantitative version of a simple geometric result. Before we state this result, recall that a maximal $r$-separated set $\mathcal{N}$ is a subset of $X$ such that for all distinct $x, y \in \mathcal{N}$ we have $d(x, y) \geq r$, and for all $z \in X$ there exists some $x \in \mathcal{N}$ with $d(z, x)<r$.

Now suppose that we are given a maximal $r$-separated set $\mathcal{N}$ in an $L$-linearly connected, $N$-doubling, complete metric space $X$. Then we can find a collection of sets $\left\{V_{x}\right\}_{x \in \mathcal{N}}$ so that each $V_{x}$ is a connected union of finitely many arcs in $X$, and for all $x, y \in \mathcal{N}$ :

(1) $d(x, y) \leq 2 r \Longrightarrow y \in V_{x}$.

(2) $\operatorname{diam}\left(V_{x}\right) \leq 5 L r$.

(3) $V_{x} \cap V_{y}=\emptyset \Longrightarrow d\left(V_{x}, V_{y}\right)>0$.

For $x \in \mathcal{N}$, we can construct each $V_{x}$ by defining it to be the union of finitely many arcs joining $x$ to each $y \in \mathcal{N}$ with $d(x, y) \leq 2 r$. By linear connectedness, we can ensure that $\operatorname{diam}\left(V_{x}\right) \leq 4 L r$. Condition (3) is trivially satisfied for compact subsets of a metric space, but we will strengthen it to the following:

$\left(3^{\prime}\right) V_{x} \cap V_{y}=\emptyset \Longrightarrow d\left(V_{x}, V_{y}\right)>\delta r$.

Lemma 3.1. We can construct the sets $V_{x}$ satisfying (1), (2) and $\left(3^{\prime}\right)$ for $\delta=$ $\delta(L, N)$.

Proof. Without loss of generality, we can rescale the metric to set $r=1$.

Since $X$ is doubling, the maximum number of 1 -separated points in a $20 \mathrm{~L}$-ball is bounded by a constant $M=M(20 L, N)$. We can label every point of $\mathcal{N}$ with an integer between 1 and $M$, such that no two points have the same label if they are separated by a distance less than $20 L$.

To find this labelling, consider the collection of all such labellings on subsets of $\mathcal{N}$ under the natural partial order. A Zorn's Lemma argument gives the existence of a maximal element: our desired labelling. So $\mathcal{N}$ is the disjoint union of $20 \mathrm{~L}$-separated $\operatorname{sets} \mathcal{N}_{1}, \ldots, \mathcal{N}_{M}$

Now let $\mathcal{N}_{\leq n}=\bigcup_{k=1}^{n} \mathcal{N}_{k}$, and consider the following:

Claim $\Delta(n)$. We can find $V_{x}$ for all $x \in \mathcal{N}_{\leq n}$, such that for all $x, y \in \mathcal{N}_{\leq n}(1),(2)$ and $\left(3^{\prime}\right)$ are satisfied with $\delta=\frac{1}{2}(5 L)^{-n}$.

$\Delta(0)$ holds trivially, and Lemma 3.1 immediately follows from $\Delta(M)$, with $\delta=$ $\delta(L, N)=\frac{1}{2}(5 L)^{-M}$. So we are done, modulo the statement that $\Delta(n) \Longrightarrow$ $\Delta(n+1)$ for $n<M$. 
Proof that $\Delta(n) \Longrightarrow \Delta(n+1)$, for $n<M$. By $\Delta(n)$, we have sets $V_{x}$ for all $x$ in $\mathcal{N}_{\leq n}$

As $\mathcal{N}_{n+1}$ is $20 L$-separated, we can treat the constructions of $V_{x}$ for each $x \in \mathcal{N}_{n+1}$ independently. We begin by creating a set $V_{x}^{(0)}$ that is the union of finitely many $\operatorname{arcs}$ joining $x$ to its 2-neighbors in $\mathcal{N}$. We can ensure that $\operatorname{diam}\left(V_{x}^{(0)}\right) \leq 4 L$.

Now construct $V_{x}^{(i)}$ inductively, for $1 \leq i \leq n . V_{x}^{(i-1)}$ can be $5 L$-close to at most one $y \in \mathcal{N}_{i}$. If $d\left(V_{x}^{(i-1)}, V_{y}\right) \in\left(0, \frac{1}{2}(5 L)^{-i}\right)$, then define $V_{x}^{(i)}$ by adding to $V_{x}^{(i-1)}$ an arc of diameter at most $L(5 L)^{-i}$ joining $V_{x}^{(i)}$ to $V_{y}$. Otherwise, let $V_{x}^{(i)}=V_{x}^{(i-1)}$. Continue until $i=n$ and set $V_{x}=V_{x}^{(n)}$.

Note that $V_{x}$ satisfies (1) and (2) by construction. The only non-trivial case we need to check for $\left(3^{\prime}\right)$ is whether $d\left(V_{x}, V_{y}\right) \in\left(0, \frac{1}{2}(5 L)^{-n}\right)$ for some $y \in \mathcal{N}_{i}$, $i<n$. (The $i=n$ case follows from the last step of the construction.) Then, since $V_{x}=V_{x}^{(n)} \supset V_{x}^{(i)}, V_{x}^{(i)} \cap V_{y} \neq \emptyset$, and $d\left(V_{x}^{(i)}, V_{y}\right) \geq \frac{1}{2}(5 L)^{-i}$. The construction then implies that

$$
\begin{aligned}
d\left(V_{x}, V_{y}\right) & \geq \frac{1}{2}(5 L)^{-i}\left(1-(2 L)(5 L)^{-1}-(2 L)(5 L)^{-2}-\cdots-(2 L)(5 L)^{-(n-i)}\right) \\
& >\frac{1}{2}(5 L)^{-n}(5 L)\left(1-\frac{2 / 5}{1-(1 /(5 L))}\right) \geq \frac{5}{2}\left(\frac{1}{2}(5 L)^{-n}\right),
\end{aligned}
$$

contradicting our assumption, so $\Delta(n+1)$ holds.

We now finish by using this construction to prove our proposition.

Proof of Proposition 2.1. By rescaling the metric, we may assume that $\iota=20 L$. If $d(a, b) \leq 20=\frac{\iota}{L}$, then join $a$ to $b$ by an arc of diameter less than $\iota$. This arc will, trivially, satisfy our conclusion for any $S \geq 1$.

Otherwise, $d(a, b)>20$. In the hypotheses for Lemma 3.1, let $r=1$ and let $\mathcal{N}$ be a maximal 1-separated set in $X$ that contains both $a$ and $b$. Now apply Lemma 3.1 to get $\left\{V_{x}\right\}_{x \in \mathcal{N}}$ satisfying (1), (2) and $\left(3^{\prime}\right)$ for $\delta=\delta(L, N)>0$.

We want to 'discretize' $A$ by finding a corresponding sequence of points in $\mathcal{N}$. Now, every open ball in $X$ meets the arc $A$ in a collection of disjoint, relatively open intervals. Since $\mathcal{N}$ is a maximal 1-separated set, the collection of open balls $\{B(x, 1): x \in \mathcal{N}\}$ covers $X$; in particular, it covers $A$. By the compactness of $A$, we can find a finite cover of $A$ by connected, relatively open intervals, each lying in some $B(x, 1), x \in \mathcal{N}$.

Using this finite cover, we can find points $x_{i} \in \mathcal{N}$ and $y_{i} \in A$ for $0 \leq i \leq n$, such that $a=y_{0}<\cdots<y_{n}=b$ in the order on $A$, and $A\left[y_{i}, y_{i+1}\right] \subset B\left(x_{i}, 1\right)$ for each $0 \leq i<n$. Since $a, b \in \mathcal{N}$, we have that $x_{0}=a$ and $x_{n}=b$. The sequence $\left(x_{0}, \ldots, x_{n}\right)$ is a discrete path in $\mathcal{N}$ that corresponds naturally to $A$.

We now find a subsequence $\left(x_{r_{j}}\right)$ of $\left(x_{i}\right)$ such that the corresponding sequence of sets $\left(V_{x_{r_{j}}}\right)$ forms a 'path' without repeats. Let $r_{0}=0$, and for $j \in \mathbb{N}^{+}$define $r_{j}$ inductively as $r_{j}=\max \left\{k: V_{x_{k}} \cap V_{x_{r_{j-1}}} \neq \emptyset\right\}$, until $r_{m}=n$ for some $m \leq n$. The integer $r_{j}$ is well defined since $d\left(y_{\left(r_{j-1}+1\right)}, x_{k}\right) \leq 1$ for $k=r_{j-1}$ and $k=r_{j-1}+1$, so $V_{x_{\left(r_{j-1}+1\right)}} \cap V_{x_{r_{j-1}}} \neq \emptyset$. Note that if $i+2 \leq j$, then $V_{x_{r_{i}}} \cap V_{x_{r_{j}}}=\emptyset$, and thus $d\left(V_{x_{r_{i}}}, V_{x_{r_{j}}}\right)>\delta$.

Let us construct our arc $J$ in segments. First, let $z_{0}=x_{r_{0}}$. Second, for each $i$ from 0 to $m-1$, let $J_{i}=J_{i}\left[z_{i}, z_{i+1}\right]$ be an arc in $V_{x_{r_{i}}}$ that joins $z_{i} \in V_{x_{r_{i}}}$ to 
some $z_{i+1} \in V_{x_{r_{i+1}}}$, where $z_{i+1}$ is the first point of $J_{i}$ to meet $V_{x_{r_{i+1}}}$. (In the case $i=m-1$, join $z_{m-1}$ to $x_{r_{m}}=z_{m}$.) Set $J=J_{0} \cup \cdots \cup J_{m}$.

This path $J$ is an arc since each $J_{i} \subset V_{x_{r_{i}}}$ is an arc, and if there exists a point $p \in J_{i} \cap J_{j}$ for some $i<j$, then $j=i+1$ and $p=z_{i+1}=z_{j}$. This is true because $V_{x_{r_{i}}} \cap V_{x_{r_{j}}} \neq \emptyset$ implies that $j=i+1$, and the definition of $z_{i+1}$ implies that $J_{i} \cap V_{x_{r_{i+1}}}=\left\{z_{i+1}\right\}$. Any finite sequence of arcs that meet only at consecutive endpoints is also an arc, so we have that $J$ is an arc.

In fact, $J$ satisfies (困). For any $y, y^{\prime} \in J, y<y^{\prime}$, we can find $i \leq j$ such that $z_{i} \leq y<z_{i+1}, z_{j} \leq y^{\prime}<z_{j+1}$. (If $y=z_{m}$, set $i=m$; likewise for $y^{\prime}$.) If $d\left(y, y^{\prime}\right) \leq \delta$, then, because $y \in V_{x_{r_{i}}}$ and $y^{\prime} \in V_{x_{r_{j}}}$, we have $d\left(V_{x_{r_{i}}}, V_{x_{r_{j}}}\right) \leq \delta$, so either $j=i$ or $j=i+1$. This gives that $J\left[y, y^{\prime}\right] \subset V_{x_{r_{i}}} \cup V_{x_{r_{j}}}$, and so $\operatorname{diam}\left(J\left[y, y^{\prime}\right]\right)$ is bounded above by $10 L$.

Furthermore, $J$-follows $A$. There is a coarse map $f: J \rightarrow A$ defined by the following composition: first map $J$ to $\mathcal{N}$ by sending $y \in J\left[z_{i}, z_{i+1}\right) \subset J$ to $x_{r_{i}} \in \mathcal{N}$, and sending $x_{r_{m}}$ to itself. Second, map each $x_{r_{i}}$ to the corresponding $y_{r_{i}}$ in $A$. Taking arbitrary $y<y^{\prime}$ in $J$ as before, we see that

$$
\begin{aligned}
J\left[y, y^{\prime}\right] & \subset J\left[z_{i}, z_{j+1}\right] \subset N\left(\left\{x_{r_{i}}, \ldots, x_{r_{j}}\right\}, 5 L\right) \subset N\left(\left\{y_{r_{i}}, \ldots, y_{r_{j}}\right\}, 5 L+1\right) \\
& \subset N\left(A\left[y_{r_{i}}, y_{r_{j}}\right], 5 L+1\right) \subset N\left(A\left[f(y), f\left(y^{\prime}\right)\right], \iota\right) .
\end{aligned}
$$

We let $s=\frac{1}{20 L} \delta$ and $S=\frac{1}{20 L} 10 L$, and have proven the proposition.

Remark. This method of proof allows one to explicitly estimate the constants given in the statements of Theorem 1.1 and Corollary [1.2, but for most applications this is not necessary.

\section{REFERENCES}

1. M. Bonk and B. Kleiner, Quasi-hyperbolic planes in hyperbolic groups, Proc. Amer. Math. Soc. 133 (2005), no. 9, 2491-2494 (electronic). MR2146190 (2005m:20098)

2. D. Burago, Y. Burago, and S. Ivanov, A course in metric geometry, Graduate Studies in Mathematics, vol. 33, American Mathematical Society, Providence, RI, 2001. MR1835418 (2002e:53053)

3. H. F. Cullen, Introduction to general topology, D. C. Heath and Co., Boston, Mass., 1968. MR0221455 (36:4507)

4. J. G. Hocking and G. S. Young, Topology, second ed., Dover Publications Inc., New York, 1988. MR:1016814(90h:54001)

5. J. M. Mackay, Spaces with conformal dimension greater than one, preprint (2007), arXiv: 0711.0417.

6. P. Tukia, Spaces and arcs of bounded turning, Michigan Math. J. 43 (1996), no. 3, 559-584. MR1420592 (98a:30028)

7. P. Tukia and J. Väisälä, Quasisymmetric embeddings of metric spaces, Ann. Acad. Sci. Fenn. Ser. A I Math. 5 (1980), no. 1, 97-114. MR595180 (82g:30038)

Department of Mathematics, University of Michigan, Ann Arbor, Michigan 481091109

Current address: Department of Mathematics, Yale University, New Haven, Connecticut 06520-8283

E-mail address: jmmackay@umich.edu 